\title{
Characterizing a Clinical Trial - Representative, Real-World Population with Heart Failure with Reduced Ejection Fraction
}

\author{
Quinn S Wells ${ }^{1,2}$ \\ Eric Farber-Eger ${ }^{1,2}$ \\ Loren Lipworth ${ }^{2,3}$ \\ Paul Dluzniewski ${ }^{4}$ \\ Ricardo Dent ${ }^{4}$ \\ John Umeijiego ${ }^{4}$ \\ Sarah S Cohen $\mathbb{D}^{3,5}$ \\ 'Division of Cardiovascular Medicine, \\ Department of Medicine, Vanderbilt \\ University Medical Center, Nashville, TN, \\ USA; ${ }^{2}$ Vanderbilt Translational and \\ Clinical Cardiovascular Research Center \\ (VTRACC), Vanderbilt University Medical \\ Center, Nashville, TN, USA; ${ }^{3}$ Division of \\ Epidemiology, Department of Medicine, \\ Vanderbilt University Medical Center, \\ Nashville, TN, USA; ${ }^{4}$ Center for \\ Observational Research, Amgen, Inc., \\ Thousand Oaks, CA, USA; \\ ${ }^{5}$ Epidstrategies, A Division of \\ Toxstrategies, Inc., Cary, NC, USA
}

Correspondence: Sarah S Cohen $\mathrm{Tel}+1$ 919-885-0548

Email scohen@epidstrategies.com
Purpose: Given known differences between real-world and clinical trial populations, we characterized demographics, clinical characteristics, and outcomes using real-world (RW) data for patients with heart failure with reduced ejection fraction (HFrEF), including those similar to subjects enrolled in an HFrEF clinical trial to better understand patient populations that could benefit from novel therapies.

Patients and Methods: Using Vanderbilt University Medical Center electronic health records (2006-2019), two RW cohorts of HFrEF patients were identified. The "Clinical Cohort" was based on a validated HFrEF algorithm and left ventricular ejection fraction (LVEF) $\leq 40 \%$. The "GALACTIC-HF-like Cohort" mirrored enrollment requirements of the GALACTIC-HF clinical trial including hospitalizations, medications, laboratory values, and LVEF $\leq 35 \%$.

Results: Median age at index for the Clinical Cohort $(\mathrm{N}=3954)$ and GALACTIC-HF-like Cohort $(\mathrm{N}=1541)$ were 65 and 61 years, respectively; both were $67 \%$ male and $80 \%$ white. Over half had coronary artery disease (55\% Clinical vs 64\% GALACTIC-HF-like); hypercholesterolemia was common (69\% Clinical vs 74\% GALACTIC-HF-like). Chronic kidney disease (31 vs 21\%), atrial fibrillation (32 vs 29\%), and cardiac resynchronization or implantable cardioverter defibrillator (26 vs 23\%) were higher in the GALACTIC-HF-like Cohort. ACE inhibitor use was high in both groups but more common in the GALACTIC-HF -like Cohort ( $71 \%$ and $82 \%$, respectively). Beta-blockers or loop diuretics were used by $>90 \%$ of both cohorts. HF hospitalization rates were 261 (95\% CI 224, 297) per 1000 person-years in Clinical versus $523(484,562)$ in GALACTIC-HF-like Cohort (median followup of 2.9 and 4.2 years, respectively).

Conclusion: Approximately $40 \%$ of RW HFrEF patients met criteria for the GALACTICHF trial. While findings of ongoing clinical trials may be directly generalizable to this sizable proportion of patients, future trials should examine whether the majority of patients with lower prevalence of comorbidities and rate of HF hospitalization could benefit from emerging HF treatments.

Keywords: heart failure, reduced ejection fraction, real-world evidence, electronic health record

\section{Plain Language Summary}

Real-world data are needed to characterize patient populations with heart failure with reduced ejection fraction ( $\mathrm{HFrEF}$ ) that could benefit from novel therapies. Using the Vanderbilt University Medical Center electronic health records, we identified a clinical cohort based on a validated HFrEF algorithm, as well as a second cohort selected to mirror 
enrollment requirements of the GALACTIC-HF clinical trial based on hospitalizations, medications, laboratory and other clinical values. Approximately $40 \%$ of RW HFrEF patients met criteria for the GALACTIC-HF trial. While findings of ongoing clinical trials may be directly generalizable to this sizable proportion of patients, whether the majority of patients with lower prevalence of comorbidities and rate of HF hospitalization could benefit from emerging HF treatments should be considered in future trials.

\section{Introduction}

Heart failure with reduced ejection fraction (HFrEF) affects at least 3 million Americans ${ }^{1,2}$ and, despite recent therapeutic advances, remains a clinical challenge, with annual mortality above $25 \%$. To date, there are no safe medical therapies that directly address the fundamental pathology of impaired cardiac contractility at the level of the cardiac sarcomere. ${ }^{3}$ The public health burden of HFrEF has made it a high priority for therapeutic development, and many new therapeutics are now being evaluated in ongoing clinical trials. ${ }^{4}$ Gauging the external validity of clinical trials can be challenging because individuals enrolled into studies may be highly selected. ${ }^{5}$ Ultimately, the potential impact of any novel therapeutic ${ }^{6}$ agent is determined by the number of patients eligible to receive it and the magnitude of the benefit provided by the therapy. Thus, it is critically important to understand the extent to which participants in trials for HFrEF reflect the patients in clinical care for whom the drug is intended, and a description of patient demographics, clinical characteristics and outcomes using real-world data of patients potentially eligible for targeted interventions and new therapeutics is needed to provide impactful real-world evidence.

Given known differences between real-world and clinical trial populations, we sought to characterize patient demographics, clinical characteristics, and outcomes using RW data for patients with $\mathrm{HFrEF}$, including those similar to subjects enrolled in an ongoing HFrEF clinical trial. Therefore, we created two real-world cohorts of HFrEF patients using a de-identified research database, the Synthetic Derivative (SD), derived from the electronic health record (EHR) of Vanderbilt University Medical Center (VUMC). The first cohort was based only on a clinical definition of HFrEF and the second cohort was based on the inclusion and exclusion criteria used in the phase-3 clinical trial (Global Approach to Lowering Adverse Cardiac outcomes Through Improving Contractility in Heart Failure, GALACTIC-HF; NCT02929329) for treatment of HFrEF.
GALACTIC-HF is a randomized, placebo-controlled, double-blind, parallel group, multicenter, cardiovascular (CV) outcomes study of omecamtiv mecarbil (OM), a first-inclass cardiac myosin activator, in subjects with $\mathrm{HFrEF}^{7}$

\section{Materials and Methods}

\section{Setting}

This study utilized information extracted from the VUMC SD database, a research tool developed to enable studies with deidentified clinical data. The SD is a de-identified copy of the VUMC EHR, and content has been transformed by deletion or permutation of all identifiers contained within each record. The SD contains nearly 3 million total records (with no defined exclusions) with highly detailed longitudinal clinical data for approximately one million subjects and an average of 27 distinct codes per record. The database incorporates data from multiple sources and includes diagnostic and procedure codes (ICD and CPT), basic demographics (age, gender, race), text from clinical care including discharge summaries, nursing notes, progress notes, history and physical, problem lists and multi-disciplinary assessments, laboratory values, electrocardiogram (ECG) diagnoses, procedural reports (eg, echocardiography), clinical text and electronically derived trace values, and inpatient medication orders. All data in the SD are updated bimonthly to append new data to clinical records of existing patients and add patients new to VUMC.

This study complies with the Declaration of Helsinki and was approved by the Vanderbilt University Medical Center Institutional Review Board. As no HIPAA identifiers are available in the Synthetic Derivative database, this study meets criteria for non-human subjects research.

\section{VUMC HF Cohort}

The Electronic Medical Records and Genomics (eMERGE) Network phenotype of HF with differentiation for preserved and reduced ejection fraction was previously developed using EHR data from the Mayo Clinic and validated in additional Mayo Clinic populations as well as within the Group Health Cooperative. ${ }^{8}$ Additional details are available online: https:// phekb.org/phenotype/heart-failure-hf-differentiation-between -preserved-and-reduced-ejection-fraction. All data elements of the eMERGE HF algorithm were previously extracted from the VUMC SD database. The ICD9 code 428.X, the structured problem list, and the unstructured problem list are the primary sources for determining classification of HF in the SD according to the eMERGE algorithm. To accommodate the discontinuation of ICD9 codes, we mapped ICD9 codes to ICD10 
codes using the CMS General Equivalence Mappings as well as clinical expertise (ICD9:428 $\leq$ ICD10:I50) and included the mapped ICD10 code in the algorithm. The structured problem list uses a controlled vocabulary that maps to SNOMED CT, whereas the unstructured problem list is free text that uses ConText, the Natural Language Processing package, to verify mentions of HF. Determination of preserved or reduced ejection fraction is based on measurements taken from transthoracic echocardiogram (TTE) reports when available. More details are available regarding the pseudocode online: https:// phekb.org/sites/phenotype/files/HF algorithm Cohort.pdf.

The adapted algorithm was validated against a set of gold standard HF subjects at VUMC. The algorithm uses the date of the qualifying HF feature (ICD code or Problem List mention) as the eMERGE definition index date ("HF index date"), as long as that date falls between an admit and discharge. Additionally, for this study, at least one TTE report was required to be available in the individual record. Thus, patients in the SD who have been diagnosed with HF according to the eMERGE Network definition and who have a TTE report made up the initial population of potentially eligible subjects for both cohorts in this study.

\section{Cohort Definitions}

Starting with the potentially eligible population of $\mathrm{HF}$ patients in the SD who met the eMERGE Network definition for HF and who had an available LVEF measurement from clinically indicated TTE, additional inclusion criteria for each cohort were applied to define the two cohorts for this study. For the Clinical Cohort, subjects were required to 1) be male or female $\geq 18$ years old and 2) have an $\mathrm{LVEF} \leq 40 \%$, using measurement closest to study index date (see the "Study Dates" section) with preference for measurements prior to index date. For the GALACTIC-HF-like Cohort, subjects were additionally required to be 1) be age 18-85 years on $\mathrm{HF}$ index date, 2) be active in the SD for at least 12 months after the HF index date, 3) have an LVEF $\leq 35 \%$, per patient's most recent TTE within the 365-day period prior to the HF index date or up to 1-month after index (if no other TTE were available prior to index), 4) have B-type natriuretic peptide (BNP) level $\geq 125 \mathrm{pg} / \mathrm{mL}$ at most recent assessment within the 365 days period prior to the HF index date, 5) have at least one written standard of care (SoC) prescription or prescription dispensed for an HFrEF medication from VUMC Pharmacy or mention on SD Medication List of standard of care HF therapies.
Standard of care HR therapies included beta blockers, angiotensin-converting enzyme inhibitors (ACEi), angiotensin receptor blockers (ARB), angiotensin receptor blocker-neprilysin inhibitor (ARNi) and mineralocorticoid receptor antagonists, and 6) have been hospitalized for a primary reason of HF or have an emergency department (ED) admission for a primary reason of HF within 1 year prior to the study index date. Subjects were excluded from the GALACTIC-HF-like Cohort if they met any of the following: 1) history of malignancy defined as any ICD code for a malignancy in the SD before the index date; 2) severe uncorrected valvular heart disease, or clinically significant congenital heart disease; 3 ) estimated glomerular filtration rate $(\mathrm{eGFR})<20 \mathrm{~mL} / \mathrm{min} / 1.73 \mathrm{~m}^{2}$ per patient's most recent medical record, within 12 months prior to study index date; 4) receiving hemodialysis within 12 months prior to study index date; 5) if available, hepatic impairment defined by a total bilirubin (TBL) $\geq 2$ times the upper limit of normal (ULN), or alanine aminotransferase $(\mathrm{ALT})$ or aspartate aminotransferase $(\mathrm{AST}) \geq 3$ times ULN within the 12 months prior to the study index date.

\section{Study Dates}

The study index date was determined for the Clinical Cohort as the eMERGE definition index date (first HF feature in the 12-month qualifying window for the eMERGE definition). This encounter could have been either an inpatient or an outpatient encounter. The index date for the GALACTIC-HF-like Cohort was selected in order to most closely mimic the entry procedures for the GALACTIC-HF trial within the existing eMERGEdefined HF cohort and thus was assigned separately for two non-overlapping groups defined by the type of encounter of their first eMERGE definition feature (hospitalization or outpatient encounter). For patients with a hospitalization as the first eMERGE definition feature, the study index date was defined as the eMERGE definition index date. For patients with an outpatient encounter as the first eMERGE definition feature, records were examined starting at the eMERGE index date moving forward in time to an $\mathrm{HF}$ hospitalization and then a subsequent HF encounter (inpatient or outpatient) occurring $\leq 365$ days after the hospitalization. This encounter date was determined to be the study index date. Study index dates were limited to occurrence within the period of 2006-2019. The baseline period was defined for both cohorts as the 1-year period prior to the index date as defined above. 


\section{Study Data}

Covariates abstracted for eligible patients were descriptive patient characteristics including demographics, comorbidities, laboratory measures (BNP, eGFR), vital signs (blood pressure and heart rate), and medication use. For each subject, all vital sign measures taken during the baseline were averaged together and this summary value was then averaged with the summary value from all other subjects. A biologic plausibility cut-off was applied to the vital signs data with values $>200$ beats per minute for heart rate being set to missing and values $>150 \mathrm{mmHg}$ for diastolic blood pressure being set to missing.

Comorbidities were obtained during the baseline period from the SD using validated algorithms of diagnostic and procedure codes developed to identify these conditions using EHR data (Supplementary Material). Presence of cardiac devices was ascertained during the baseline period as well as in any existing records prior to the baseline period in order to more accurately count patients who had ever received a device. Laboratory measures were also obtained during the baseline period. Medications were obtained from the SD as Yes/No to ever use. Dispensed prescription data from a VUMC Pharmacy are contained directly within an individual's SD record. Additional sources within the SD routinely used for identification of medication use include clinical documents, outpatient and inpatient orders, medications administered during inpatient care, problem lists, medical histories, and discharge notes; within these SD sources, data on medication use are contained as written prescriptions, list of inpatient medications administered, medication list entries, or patient selfreported medication (identified from notes and read by NLP program "MedEx"). ${ }^{9}$

Outcomes assessed during follow-up included HF hospitalization, and death when available. Follow-up for each cohort for HF hospitalization or death began on the study index date and continued until the patient's last SD encounter or date of death, whichever occurred first. Follow-up in the SD was available through April 30, 2020. Complete ascertainment of death, however, was available only through February 1, 2017, so for analyses of death as the outcome, follow-up was truncated for all participants on that date.

\section{Statistical Methods}

All analyses were descriptive in nature. Categorical variables were described in terms of sample sizes (N) and percentages (\%). Continuous variables were described in terms of mean, median, standard deviation, minimum, and maximum. Rates of HF hospitalization were calculated as incidence rates from 3 days post $\mathrm{HF}$ index date to last $\mathrm{SD}$ encounter or death and presented as number of events per 1000 person-years. Three days was chosen after considering a range of censoring windows (ie, days after index date before events were captured) in order to evaluate the balance of avoiding the index event in the counts while capturing most events during follow-up. Incidence rates included only the first HF hospitalization captured during follow-up.

\section{Results}

The eMERGE Network definition of HF was applied to the $>3$ million records in the SD and 38,668 patients met the definition for HF (Figure 1). Restricting to those with a transthoracic echocardiogram-derived ejection fraction resulted in 27,586 subjects, which was further reduced to 5488 when subjects with a preserved ejection fraction and those with no ejection fraction before their index date were excluded. Restricting to those with an index date from 1/1/ 2006 to $12 / 31 / 2019$ as well as the requirements of the Clinical Cohort resulted in 3954 subjects. These patients were then evaluated against the criteria for the GALACTIC-HF-like Cohort and grouped accordingly, with 3954 patients in the Clinical Cohort and the subgroup of those $(\mathrm{N}=1541)$ meeting criteria for inclusion in the GALACTIC-HF-like Cohort.

Descriptive characteristics of the two cohorts are shown in Table 1. The Clinical Cohort was older than the GALACTIC-HF-like Cohort at index (median 64.6 versus 60.5 years). Both cohorts were two-thirds male. The Clinical Cohort was $80.7 \%$ White compared with $77.4 \%$ in the GALACTIC-HF-like Cohort. As a result of the study design which required patients in the GALACTIC-HF-like Cohort to be active in the SD for at least 12 months after the HF index date, the minimum follow-up for this cohort is 1 year. In the Clinical Cohort, there was no such restriction and thus the minimum follow-up could be as short as a single day. This resulted in a longer median follow-up time for the GALACTIC-HFlike Cohort (median 4.2 years, range 1-13.6) versus the Clinical Cohort (median 2.9 years, range $0-13.8$ ).

Clinical characteristics of the two cohorts, including vital signs and laboratory measurements, are summarized in Table 1. Heart rate was higher on average in the GALACTIC-HF-like Cohort compared with the Clinical Cohort (median 82 vs 77 bpm). Both BMI and diastolic 


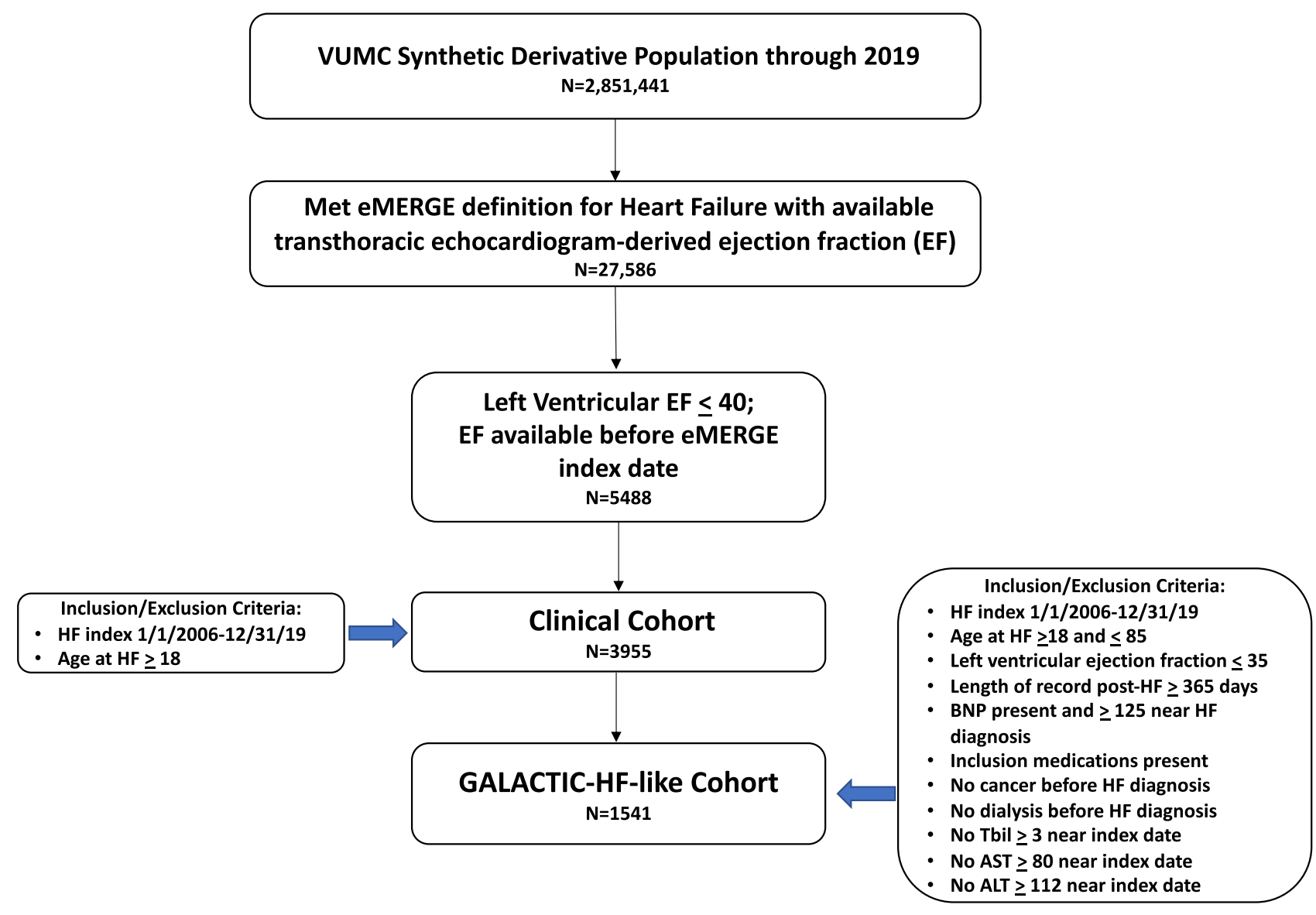

Figure I Cohort flow diagram.

blood pressure was similar between the two groups, whereas systolic blood pressure was slightly lower in the GALACTIC-HF-like Cohort (115 vs $121 \mathrm{mmHg}$ ). Mean BNP values were higher in the GALACTIC-HF-like Cohort than in the Clinical Cohort (820.8 vs 506.0 pg/ $\mathrm{mL}$ ), but eGFR values were similar in both cohorts. The median LVEF in the Clinical Cohort was 30\% versus $22.5 \%$ in the GALACTIC-HF-like Cohort, a difference at least partially due to the study design, which utilized different cut-offs for inclusion in each cohort (LVEF $\leq 40 \%$ for Clinical and $\leq 35 \%$ for GALACTIC-HF-like).

Table 2 provides a summary of comorbidity prevalence and use of cardiac devices in the cohorts during the baseline period. Generally, comorbidities were somewhat more common in the GALACTIC-HF-like Cohort than in the Clinical Cohort although this should be interpreted with caution given that the GALACTIC-HF-like Cohort had a minimum of 1 year and overall longer mean follow-up than the Clinical Cohort by design. More than half of the subjects had coronary artery disease $(55 \%$ of the clinical and $64 \%$ of the GALACTIC-HF-like Cohort), and hypercholesterolemia was common $(69 \%$ in the Clinical and $74 \%$ in the GALACTIC-HF-like Cohorts). Chronic kidney disease (31 vs 21\%), atrial fibrillation (32 vs $29 \%$ ), and cardiac resynchronization or implantable cardioverter defibrillator (26 vs $23 \%$ ) were higher in the GALACTIC-HF-like Cohort.

Table 3 displays the prevalence of use of medications for HFrEF and selected other chronic conditions during the one-year baseline period by cohort. With respect to commonly used HFrEF treatments, use of an ACE inhibitor was high in both groups but more common in the GALACTIC-HF-like Cohort (71\% and $82 \%$, respectively), and beta-blockers or loop diuretics were used by over $90 \%$ of the individuals in both cohorts. Mineralocorticoid receptor antagonists (55\% and 77\%, respectively) and $\mathrm{ARBs}(39 \%$ and $46 \%$, respectively) were used somewhat less frequently, but a pattern of higher use in the GALACTIC-HF-like Cohort compared to the Clinical Cohort was apparent also for these medications. Over $75 \%$ of the patients in both groups were prescribed statins. 
Table I Demographic and Clinical Characteristics of Clinical and GALACTIC-HF-Like Cohorts ${ }^{\mathrm{a}}$

\begin{tabular}{|c|c|c|}
\hline & Clinical Cohort $N=3954$ & GALACTIC-HF-Like Cohort $\mathrm{N}=|54|$ \\
\hline Age at index, years & $64.6[54.4,73.8]$ & $60.5[50.5,69.2]$ \\
\hline$<65$ & $2016(51.0 \%)$ & $977(63.4 \%)$ \\
\hline$\geq 65$ & $1052(26.6 \%)$ & $370(24.0 \%)$ \\
\hline$\geq 75$ & $887(22.4 \%)$ & $194(12.6 \%)$ \\
\hline \multicolumn{3}{|l|}{ Sex } \\
\hline Male & $2652(67.1 \%)$ & 1044 (67.7\%) \\
\hline Female & 1302 (32.9\%) & $497(32.3 \%)$ \\
\hline \multicolumn{3}{|l|}{ Race/Ethnicity } \\
\hline White & $3190(80.7 \%)$ & II 93 (77.4\%) \\
\hline Black & $624(15.8 \%)$ & $306(19.9 \%)$ \\
\hline Asian & $24(0.6 \%)$ & $8(0.5 \%)$ \\
\hline American Indian/Native Alaskan & $6(0.2 \%)$ & $2(0.1 \%)$ \\
\hline Other & $6(0.1 \%)$ & $4(0.3 \%)$ \\
\hline Unknown & $104(2.6 \%)$ & $28(1.8 \%)$ \\
\hline Body mass index $\left(\mathrm{kg} / \mathrm{m}^{2}\right)^{\mathrm{b}}$ & $28.7[24.9,33.3]$ & $28.5[24.9,32.9]$ \\
\hline Follow-up time in SD, years & $\begin{array}{c}\text { Median: } 2.9 \\
\text { IQR }[1.2,5.3]\end{array}$ & $\begin{array}{c}\text { Median: } 4.2^{\mathrm{C}} \\
\text { IQR }[2.7,6.3]\end{array}$ \\
\hline LVEF closest to index date & $\begin{array}{c}\text { Median: } 30.0 \\
\text { IQR }[21.0,35.0]\end{array}$ & $\begin{array}{c}\text { Median: } 22.5 \\
\text { IQR }[15.0,30.0]\end{array}$ \\
\hline \multicolumn{3}{|c|}{ Vital Signs } \\
\hline Systolic blood pressure $(\mathrm{mmHg})^{b}$ & $|2|[\mid 10.5,133.8]$ & $115[103.8,129.1]$ \\
\hline Diastolic blood pressure $(\mathrm{mmHg})^{\mathrm{b}}$ & $70[64.0,78.0]$ & $70[63.2,77.9]$ \\
\hline Heart rate (beats per minute) ${ }^{b}$ & $77[69.5,87.7]$ & $82[72.4,94.0]$ \\
\hline \multicolumn{3}{|c|}{ Laboratory measurements } \\
\hline BNP (pg/mL) & $506.0[191.0,1164.0]$ & $820.8[42|.1| 5 \mid 3.8]$, \\
\hline Number of measurements in record during baseline period & $\mathrm{I} .0[0.0, \mathrm{I} .0]$ & $\mathrm{I} .0[1.0,2.0]$ \\
\hline eGFR (mL/min/l.73m²) & $66.0[48.1,83.4]$ & $64.7[48.2,81.3]$ \\
\hline Number of measurements in record during baseline period & $2.0[1.0,4.0]$ & $3.0[1.0,9.0]$ \\
\hline
\end{tabular}

Notes: ${ }^{\text {a }}$ alues expressed at $\mathrm{N}(\%)$ for categorical variables and median (25th, 75 th percentile) for continuous variables. ${ }^{b}$ Median of the mean of all measures taken during baseline period was used for each subject. For body mass index, median of the median was used. In order to more closely mimic the clinical trial structure, the criteria for meeting the GALACTIC-HF-like Cohort definition included the requirement of being active in the SD for at least 12 months after the HF index date.

Event rates are displayed in Figure 2 for HF hospitalizations and deaths per 1000 person-years. Both heart failure hospitalization rates and death rates were higher in the GALACTIC-HF-like Cohort compared with the Clinical Cohort. With a 3-day post-index censoring window, HF hospitalization rates were 261 (95\% CI 224, 297) per
1000 person-years in the Clinical cohort versus 523 (484, 562) in the GALACTIC-HF-like Cohort, during median follow-up of 2.9 and 4.2 years, respectively. Death rates were $260(207,313)$ and $277(94,460)$ per 1000 personyears in the Clinical Cohort and GALACTIC-HF-like Cohort, respectively. 
Table 2 Proportion of Individuals with Select Comorbidities and Cardiac Devices at Baseline in Clinical and GALACTIC-HF-Like Cohorts

\begin{tabular}{|c|c|c|}
\hline & Clinical Cohort N=3954 & GALACTIC-HF-Like Cohort $N=|54|$ \\
\hline & $\mathbf{N}$ with I+ instances in baseline period (\%) & $\mathbf{N}$ with I+ instances in baseline period (\%) \\
\hline Coronary artery disease & $2167(54.8 \%)$ & $984(63.9 \%)$ \\
\hline Myocardial infarction & $660(16.7 \%)$ & $282(18.3 \%)$ \\
\hline Unstable angina & $312(7.9 \%)$ & $172(11.2 \%)$ \\
\hline Stable angina & $216(5.5 \%)$ & $80(5.2 \%)$ \\
\hline Stroke & $579(14.6 \%)$ & $286(18.6 \%)$ \\
\hline Transient ischemic attack & $72(1.8)$ & $29(1.9 \%)$ \\
\hline Revascularization & 1088 (27.5\%) & 577 (37.4\%) \\
\hline Valve disease & $199(5.0 \%)$ & $96(6.2 \%)$ \\
\hline Atrial fibrillation or flutter & II 32 (28.6\%) & 485 (31.5\%) \\
\hline Peripheral artery disease & $159(4.0 \%)$ & $78(5.1 \%)$ \\
\hline Chronic obstructive pulmonary disease & $306(7.7 \%)$ & 154 (10.0\%) \\
\hline Chronic kidney disease & $818(20.7 \%)$ & $470(30.5 \%)$ \\
\hline Diabetes mellitus & $946(23.9 \%)$ & $413(26.8 \%)$ \\
\hline Hypercholesterolemia & 2739 (69.3\%) & II 34 (73.6\%) \\
\hline Any CRT or ICD & 915 (23.1\%) & 396 (25.7\%) \\
\hline
\end{tabular}

Abbreviations: CRT, cardiac resynchronization therapy; ICD, implantable cardioverter defibrillator.

\section{Discussion}

A clear understanding of patient demographics, clinical characteristics, and outcomes among HFrEF patients from a realworld data resource is critical to define patient populations that may benefit from novel HFrEF therapies. In this study, conducted within the VUMC EHR database, nearly 4000 patients were identified who met a validated clinical definition of $\mathrm{HFrEF}^{8}$ between 2006 and 2019. A subset of approximately $40 \%$ of these patients met the more stringent inclusion criteria of the GALACTIC-HF trial. ${ }^{6}$ This finding suggests that results of current clinical trials for new therapeutics may be directly generalizable to a sizable proportion of real-world HFrEF patients in clinical practice. However, whether the remaining majority of HFrEF patients with lower prevalence of comorbidities and lower rate of HF hospitalization could benefit from emerging HF treatments should be considered in future trials.

Although this analysis was conducted in a single quaternary care hospital with a high-volume HF and transplantation program geographically located in a region with a high burden of HF, the findings, including demographic and clinical characteristics, are largely in agreement with published baseline data from GALACTIC-HF and other clinical trial ${ }^{6,10,11}$ and community populations, ${ }^{12}$ such as the global PARADIGM-HF trial, the VICTORIA trial, and the recent PCORnet publication and are likely relevant to the broader HF population. ${ }^{10,13}$ Of note is the general comparability of baseline characteristics between the GALACTIC-HF-like Cohort in our study and the GALACTIC-HF trial participants, ${ }^{6}$ including mean age (61 vs 65 years, respectively), race (77 vs $78 \%$ white), prevalence of coronary artery disease (64 vs $62 \%$ ), and median eGFR ( 65 vs $59 \mathrm{~mL} / \mathrm{min} / 1.73 \mathrm{~m}^{2}$ ). Prevalence of certain comorbidities such as atrial fibrillation/flutter and diabetes was lower in our GALACTIC-HF-like Cohort than in the GALACTIC-HF trial, and there was a higher proportion of women in our cohort (32 vs $21 \%$ ).

Although specific practice patterns and use of evidence-based therapies are known to vary by geographic region or disease severity and across clinical settings, goal-directed medical therapy, including ACE inhibitors, beta blockers, and mineralocorticoid receptor antagonists, 
Table 3 Prevalence of Targeted Medications Post-Ascertainment in Clinical and GALACTIC-HF-Like Cohorts

\begin{tabular}{|c|c|c|}
\hline Medication Type & $\begin{array}{c}\text { Clinical Cohort } \\
\qquad N=3954\end{array}$ & $\begin{array}{l}\text { GALACTIC-HF-Like } \\
\text { Cohort } N=|54|\end{array}$ \\
\hline Beta blockers & 3738 (94.5\%) & 1516 (98.4\%) \\
\hline ACE inhibitors & $2813(71.1 \%)$ & 1277 (82.9\%) \\
\hline ARBs & 1537 (38.9\%) & 707 (45.9\%) \\
\hline Loop diuretics & 3501 (88.5\%) & 1514 (98.2\%) \\
\hline ARNis & $383(9.7 \%)$ & $162(10.5 \%)$ \\
\hline $\begin{array}{l}\text { Mineralocorticoid } \\
\text { receptor antagonists }\end{array}$ & $2183(55.2 \%)$ & $1186(77.0 \%)$ \\
\hline Antiarrhythmics & $1613(40.8 \%)$ & $818(53.1 \%)$ \\
\hline $\begin{array}{l}\text { Platelet aggregation } \\
\text { inhibitors }\end{array}$ & $3428(86.7 \%)$ & 1452 (94.2\%) \\
\hline $\begin{array}{l}\text { Other antithrombotics/ } \\
\text { Anticoagulants }\end{array}$ & $2169(54.9 \%)$ & $1021(66.3 \%)$ \\
\hline Renin inhibitors & $12(0.3 \%)$ & $6(0.4 \%)$ \\
\hline Digitalis glycosides & 978 (24.7\%) & $52 \mid(33.8 \%)$ \\
\hline Thiazide and thiazide-like & 1077 (27.2\%) & $572(37.1 \%)$ \\
\hline Oral nitrates & $979(24.8 \%)$ & $497(32.3 \%)$ \\
\hline $\begin{array}{l}\text { Hydralazine and oral } \\
\text { nitrates }\end{array}$ & $36(0.9 \%)$ & $20(1.3 \%)$ \\
\hline Ivabradine & $25(0.6 \%)$ & $13(0.8 \%)$ \\
\hline \multicolumn{3}{|c|}{ Cholesterol-lowering medications } \\
\hline Statins & 3063 (77.5\%) & 1300 (84.4\%) \\
\hline Ezetimibe & $328(8.3 \%)$ & $172(11.2 \%)$ \\
\hline PCSK9 inhibitors & $47(1.2 \%)$ & $7(0.5 \%)$ \\
\hline \multicolumn{3}{|l|}{ Diabetes medications } \\
\hline Insulin & 1757 (44.4\%) & $862(55.9 \%)$ \\
\hline Sulfonylureas & $701(17.7 \%)$ & $349(22.6 \%)$ \\
\hline SGLT2 inhibitors & $104(2.6 \%)$ & $50(3.2 \%)$ \\
\hline Metformin & $812(20.5 \%)$ & $376(24.4 \%)$ \\
\hline $\begin{array}{l}\text { Alpha glucosidase } \\
\text { inhibitors }\end{array}$ & $4(0.1 \%)$ & $2(0.1 \%)$ \\
\hline Meglitinides & $20(0.5 \%)$ & $12(0.8 \%)$ \\
\hline
\end{tabular}

Abbreviations: ARB, angiotensin receptor blockers; ACEi, angiotensin-converting enzyme inhibitors; ARNi, angiotensin receptor blocker-neprilysin inhibitor; SGLT2, sodium-glucose transport protein 2 .

has been the cornerstone of HFrEF therapy for decades. This approach follows consistent evidence from trials showing a reduced risk of death and/or hospitalization due to HF associated with use of these medications. ${ }^{14-19}$ As expected, the observed patterns of medication use in the two real-world cohorts in our study are consistent with this approach to HFrEF treatment, showing high prevalence of use of ACE inhibitors and beta-blockers in both cohorts, in line with evidence that, when used together with ACE inhibitors, beta-blockers are associated with incremental decreases in the risk of death ${ }^{14,20-23}$ among HFrEF patients. Use of mineralocorticoid receptor antagonists was somewhat higher in the GALACTIC-HF-like Cohort, which may be an indicator of its predominant use in sicker HFrEF patients who are taking other drugs known to improve outcomes. ${ }^{22}$ In our study cohorts, with enrollment from 2006-2019, the low prevalence of use of SGLT2 inhibitors $(<3 \%)$ is likely a reflection of their recent approval for reduction of cardiovascular death and HF hospitalization among $\mathrm{HFrEF}^{24-27}$ patients with and without Type 2 diabetes. Nevertheless, during the vast majority of the study period, there were no novel agents introduced to escalate care for HFrEF patients experiencing clinically-relevant outcomes, underscoring the need for novel therapeutics for HFrEF and for future work to determine uptake of new therapies and potential interventions that may be needed to increase utilization of therapies for which efficacy is demonstrated.

Clinical trials often enroll higher risk patient populations where power is greatest to detect beneficial effects of novel therapies. Indeed, subjects included in the GALACTIC-HF, and other recent trials, often have highrisk features including current/recent hospitalization, elevated BNP levels, and severely depressed LV function. The fact that $40 \%$ of our HFrEF patients met these inclusion criteria reinforces the high burden of severe HF among patients in clinical care. Moreover, the high rate of HF hospitalization in both the Clinical Cohort and GALACTIC-HF-like Cohort in our study, despite the use of standard medical therapy for HF, indicate that these patients have residual risk and contribute to the high healthcare burden of HF.

Our analysis has limitations that should be acknowledged. While we believe our findings are relevant more broadly, this was a single-center study and there may be aspects of our patient population that reduce the generalizability of our results. For example, individuals in our study were predominantly white, and results should be replicated in populations with different racial composition. Additionally, referral and practice patterns may differ 


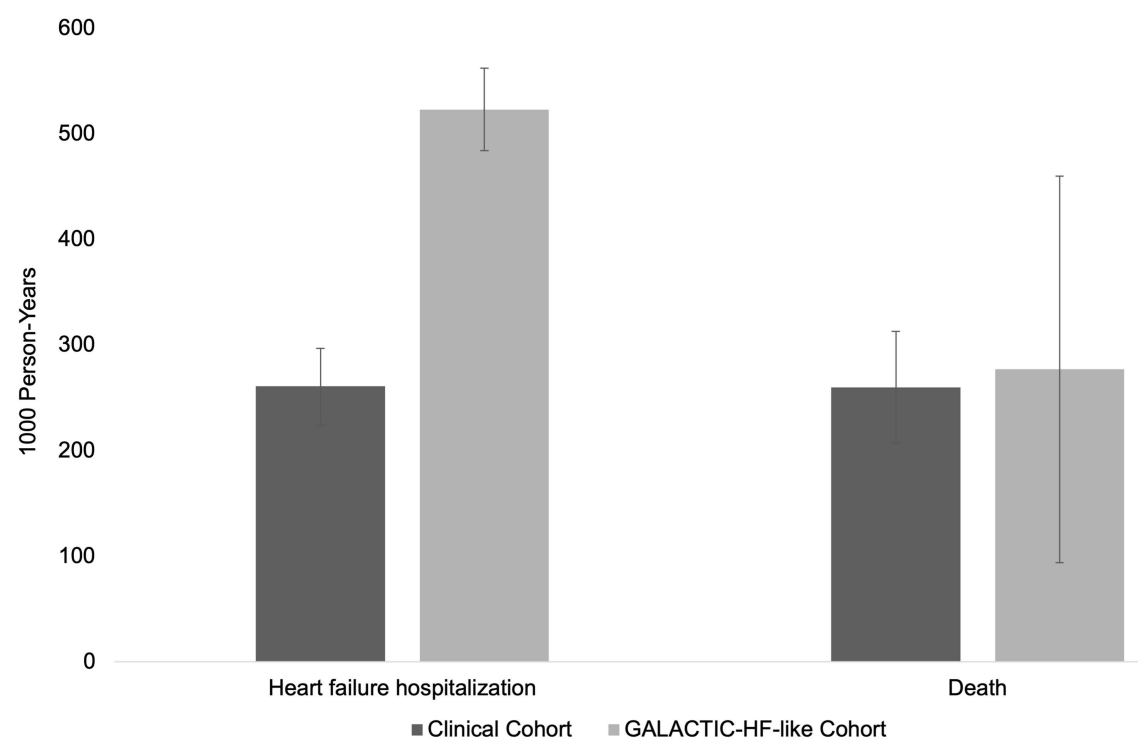

Figure 2 Heart failure hospitalizations and deaths per 1000 person-years in Clinical and GALACTIC-HF-like Cohorts. Footnote: Bars show events per I000 person-years; whiskers show $95 \%$ confidence intervals. There were a total of 2117 HF hospitalizations in the Clinical Cohort and II80 in the GALACTIC-HF-like Cohort. There were a total of 1019 deaths in the Clinical Cohort and 379 in the GALACTIC-HF-like Cohort.

across institutions, which could affect the generalizability of our findings. It is also possible that some data elements were under-ascertained due to care encounters at other institutions. However, the substantial follow-up time in our study should significantly attenuate underascertainment by assuring that subjects had sufficient opportunities for comorbidity documentation. Additionally, although misclassification related to errors in EHR documentation is possible, we addressed this weakness through the use of validated phenotype algorithms. As comprehensive mortality linkages to the SD were current only through 2017, the analysis of death rates was limited to follow-up through 2017 only. Finally, while mirrored as closely as possible in our dataset, we were unable to perfectly match GALACTIC-HF trial inclusion and exclusion criteria.

\section{Conclusions}

Our study highlights additional aspects of HFrEF patients in the real world. Although a substantial number of patients were similar to clinical trial populations, and accounted for a larger number of adverse outcomes, over half of the HFrEF patients did not meet GALACTIC-HF trial inclusion criteria ${ }^{6}$ although they still experienced high rates of HF hospitalizations and deaths. It is encouraging that results of ongoing trials will likely be relevant to a large number of high-risk HFrEF patients; however, the extent to which they will be generalizable to all HFrEF patients is less clear and may need to be addressed in future trials that enroll patients more broadly across the spectrum of HFrEF severity.

\section{Abbreviations}

$\mathrm{ARB}$, angiotensin receptor blockers; ACEi, angiotensinconverting enzyme inhibitors; ALT, alanine aminotransferase; AST, aspartate aminotransferase; ARNi, angiotensin receptor blocker-neprilysin inhibitor; BNP, B-type natriuretic peptide; CV, cardiovascular; ECG, electrocardiogram; ED, emergency department; eGFR, estimated glomerular filtration rate; EHR, electronic health record; eMERGE, Electronic Medical Records and Genomics; GALACTIC-HF, Global Approach to Lowering Adverse Cardiac outcomes Through Improving Contractility in Heart Failure; HFrEF, heart failure with reduced ejection fraction; LVEF, left ventricular ejection fraction; OM, omecamtiv mecarbil; RW, real world; SD, synthetic derivative; SoC, standard of care; TBL, total bilirubin; TTE, transthoracic echocardiogram; VUMC, Vanderbilt University Medical Center; ULN, upper limit of normal.

\section{Data Sharing Statement}

Vanderbilt University Medical Center is firmly committed to sharing data with the scientific community so that the data generated from this study can be fully utilized for research. VUMC also has an obligation to protect the privacy of study participants and the confidentiality of study data, since this project is an ancillary study of the Vanderbilt Synthetic Derivative, which has guidelines in place that enable 
scientific investigators to apply for use of these data. The Vanderbilt BioVU public availability is described on the VICTR website: https://victr.vumc.org/overview-ofresources/. Aggregated statistics will be provided to the broad scientific community via the journal's web site or to an individual investigator/team upon request.

\section{Author Contributions}

All authors made a significant contribution to the work reported, whether that is in the conception, study design, execution, acquisition of data, analysis and interpretation, or in all these areas; took part in drafting, revising or critically reviewing the article; gave final approval of the version to be published; have agreed on the journal to which the article has been submitted; and agree to be accountable for all aspects of the work.

\section{Funding}

This work was supported by a research grant to EpidStrategies from Amgen, Inc., Thousand Oaks, CA, USA. The project was also supported by the National Center for Research Resources, Grant UL1 RR02497501, and is now at the National Center for Advancing Translational Sciences, Grant 2 UL1 TR000445-06. The content is solely the responsibility of the authors and does not necessarily represent the official views of the NIH.

\section{Disclosure}

Paul Dluzniewski, Ricardo Dent, and John Umeijiego are employees and stockholders of Amgen, Inc. Sarah $\mathrm{S}$ Cohen is an employee of EpidStrategies, A D ivision of ToxStrategies, Inc., who received research funds from Amgen, Inc. All other authors have no financial or nonfinancial interests to declare.

\section{References}

1. Virani SS, Alonso A, Benjamin EJ, et al. Heart disease and stroke statistics-2020 update: a report from the American Heart Association. Circulation. 2020;141(9):e139-e596. doi:10.1161/CIR.000000 0000000757

2. Shah KS, Xu H, Matsouaka RA, et al. Heart failure with preserved, borderline, and reduced ejection fraction: 5-year outcomes. $J$ Am Coll Cardiol. 2017;70(20):2476-2486. doi:10.1016/j.jacc.2017.08.074

3. Malik FI, Hartman JJ, Elias KA, et al. Cardiac myosin activation: a potential therapeutic approach for systolic heart failure. Science. 2011;331(6023):1439-1443. doi:10.1126/science.1200113

4. Kish T. New heart failure medications aim to fill significant gaps in treatment. $P$ T. 2017;42(12):764-766.

5. Rothwell PM. External validity of randomised controlled trials: "to whom do the results of this trial apply?". Lancet. 2005;365 (9453):82-93. doi:10.1016/S0140-6736(04)17670-8
6. Teerlink JR, Diaz R, Felker GM, et al. Omecamtiv mecarbil in chronic heart failure with reduced ejection fraction: GALACTIC-HF baseline characteristics and comparison with contemporary clinical trials. Eur J Heart Fail. 2020;22(11):2160-2171.

7. Kaplinsky E, Mallarkey G. Cardiac myosin activators for heart failure therapy: focus on omecamtiv mecarbil. Drugs Context. 2018;7:212518. doi:10.7573/dic.212518

8. Bielinski SJ, Pathak J, Carrell DS, et al. A robust e-Epidemiology Tool in phenotyping heart failure with differentiation for preserved and reduced ejection fraction: the Electronic Medical Records and Genomics (eMERGE) network. $J$ Cardiovasc Transl Res. 2015;8 (8):475-483. doi:10.1007/s12265-015-9644-2

9. Xu H, Stenner SP, Doan S, Johnson KB, Waitman LR, Denny JC MedEx: a medication information extraction system for clinical narratives. J Am Med Inform Assoc. 2010;17(1):19-24.

10. McMurray JJ, Packer M, Desai AS, et al. Angiotensin-neprilysin inhibition versus enalapril in heart failure. $N$ Engl J Med. 2014;371 (11):993-1004. doi:10.1056/NEJMoa1409077

11. McMurray JJV, Solomon SD, Inzucchi SE, et al. Dapagliflozin in patients with heart failure and reduced ejection fraction. $N$ Engl J Med. 2019;381(21):1995-2008. doi:10.1056/NEJMoa1911303

12. Mentz RJ, Xu H, O’Brien EC, et al. PROVIDE-HF primary results: patient-reported outcomes investigation following initiation of drug therapy with Entresto (sacubitril/valsartan) in heart failure. Am Heart J. 2020;230:35-43. doi:10.1016/j.ahj.2020.09.012

13. Armstrong PW, Pieske B, Anstrom KJ, et al. Vericiguat in patients with heart failure and reduced ejection fraction. $N$ Engl $J$ Med. 2020;382(20):1883-1893. doi:10.1056/NEJMoa1915928

14. Packer M, Fowler MB, Roecker EB, et al. Effect of carvedilol on the morbidity of patients with severe chronic heart failure: results of the carvedilol prospective randomized cumulative survival (COPERNICUS) study. Circulation. 2002;106(17):2194-2199. doi:10.1161/01.CIR.0000035653.72855.BF

15. CONSENSUS Trial Study Group. Effects of enalapril on mortality in severe congestive heart failure. Results of the Cooperative North Scandinavian Enalapril Survival Study (CONSENSUS). $N$ Engl $J \quad$ Med. 1987;316(23):1429-1435. doi:10.1056/NEJM19870604 3162301

16. Hjalmarson A, Goldstein S, Fagerberg B, et al. Effects of controlled-release metoprolol on total mortality, hospitalizations, and well-being in patients with heart failure: the Metoprolol CR/XL randomized intervention trial in congestive heart failure (MERIT-HF). MERIT-HF Study Group. JAMA. 2000;283 (10):1295-1302. doi:10.1001/jama.283.10.1295

17. Yusuf S, Pitt B, Davis CE, Hood WB, Cohn JN. Effect of enalapril on survival in patients with reduced left ventricular ejection fractions and congestive heart failure. N Engl J Med. 1991;325(5):293-302.

18. Rossello X, Ariti C, Pocock SJ, et al. Impact of mineralocorticoid receptor antagonists on the risk of sudden cardiac death in patients with heart failure and left-ventricular systolic dysfunction: an individual patient-level meta-analysis of three randomized-controlled trials. Clin Res Cardiol. 2019;108(5):477-486. doi:10.1007/s00392-018$1378-0$

19. Wikstrand J, Wedel H, Castagno D, McMurray JJ. The large-scale placebo-controlled beta-blocker studies in systolic heart failure revisited: results from CIBIS-II, COPERNICUS and SENIORS-SHF compared with stratified subsets from MERIT-HF. J Intern Med. 2014;275(2):134-143. doi:10.1111/joim.12141

20. MERIT-HF. Effect of metoprolol CR/XL in chronic heart failure: metoprolol $\mathrm{CR} / \mathrm{XL}$ randomised intervention trial in congestive heart failure (MERIT-HF). Lancet. 1999;353(9169):2001-2007. doi:10.1016/S0140-6736(99)04440-2

21. CIBIS-II. The cardiac insufficiency Bisoprolol study II (CIBIS-II): a randomised trial. Lancet. 1999;353(9146):9-13. doi:10.1016/ S0140-6736(98)11181-9 
22. Pitt B, Zannad F, Remme WJ, et al. The effect of spironolactone on morbidity and mortality in patients with severe heart failure. Randomized Aldactone Evaluation Study Investigators. $N$ Engl J Med. 1999;341(10):709-717. doi:10.1056/NEJM199909023411001

23. Zannad F, McMurray JJ, Krum H, et al. Eplerenone in patients with systolic heart failure and mild symptoms. $N$ Engl J Med. 2011;364 (1):11-21. doi:10.1056/NEJMoa1009492

24. Neal B, Perkovic V, Mahaffey KW, et al. Canagliflozin and cardiovascular and renal events in type 2 diabetes. $N$ Engl J Med. 2017;377 (7):644-657. doi:10.1056/NEJMoa1611925
25. Perkovic V, Jardine MJ, Neal B, et al. Canagliflozin and renal outcomes in type 2 diabetes and nephropathy. $N$ Engl J Med. 2019;380 (24):2295-2306. doi:10.1056/NEJMoa1811744

26. Wiviott SD, Raz I, Bonaca MP, et al. Dapagliflozin and cardiovascular outcomes in type 2 diabetes. $N$ Engl J Med. 2019;380 (4):347-357. doi:10.1056/NEJMoa1812389

27. Zinman B, Wanner C, Lachin JM, et al. Empagliflozin, cardiovascular outcomes, and mortality in type 2 diabetes. $N$ Engl J Med. 2015;373(22):2117-2128. doi:10.1056/NEJMoa1504720

\section{Publish your work in this journal}

Clinical Epidemiology is an international, peer-reviewed, open access, online journal focusing on disease and drug epidemiology, identification of risk factors and screening procedures to develop optimal preventative initiatives and programs. Specific topics include: diagnosis, prognosis, treatment, screening, prevention, risk factor modification,

Submit your manuscript here: https://www.dovepress.com/clinical-epidemiology-journal systematic reviews, risk \& safety of medical interventions, epidemiology \& biostatistical methods, and evaluation of guidelines, translational medicine, health policies \& economic evaluations. The manuscript management system is completely online and includes a very quick and fair peer-review system, which is all easy to use. 